
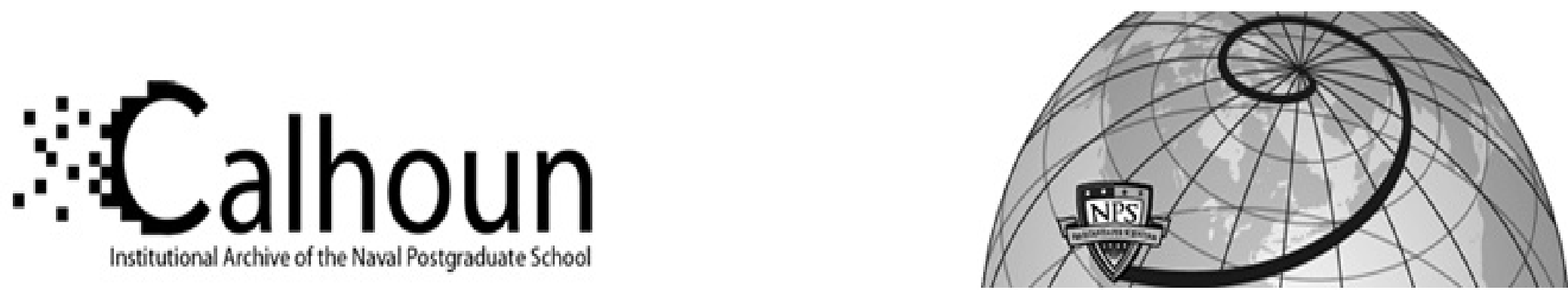

Calhoun: The NPS Institutional Archive DSpace Repository

\title{
On the Prediction of Dynamic Stall Onset on Airfoils in Low Speed Flow
}

Jones, K.D.; Platzer, M.F.

Jones, K.D. and Platzer, M.F., "On the Prediction of Dynamic Stall Onset on Airfoils in Low Speed Flow," Proceedings of the 8th International Symposium on Unsteady Aerodynamics and Aeroelasticity of Turbomachines, Ed. T. H. Fransson, Kluwer Academic Publications, Dordrecht, The Netherlands, 1998, pp. 797-812.

https://hdl.handle.net/10945/37219

This publication is a work of the U.S. Government as defined in Title 17, United States Code, Section 101. Copyright protection is not available for this work in the United States.

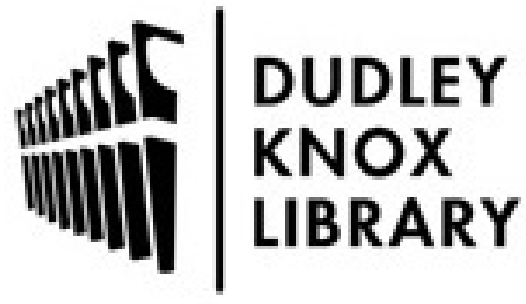

http://www.nps.edu/library
Calhoun is the Naval Postgraduate School's public access digital repository for research materials and institutional publications created by the NPS community. Calhoun is named for Professor of Mathematics Guy K. Calhoun, NPS's first appointed -- and published -- scholarly author.

Dudley Knox Library / Naval Postgraduate School 411 Dyer Road / 1 University Circle Monterey, California USA 93943 


\title{
ON THE PREDICTION OF DYNAMIC STALL ONSET ON AIR- FOILS IN LOW SPEED FLOW
}

\author{
K. D. JONES and M. F. PLATZER \\ Naval Postgraduate School \\ Code AA/Jo, 699 Dyer Rd., Monterey, CA 93943-5106
}

\section{Abstract}

A computational approach is described for the rapid and systematic prediction and evaluation of the onset of dynamic stall due to rapid incidence changes or unsteady pitch or plunge motions. The method combines an unsteady, two-dimensional panel code with a two-dimensional boundary-layer code. The panel code provides incompressible, inviscid flowfields about arbitrary airfoils undergoing prescribed motions. The boundary-layer code computes laminar, transitional and turbulent regimes.

Presented results demonstrate that the delay in dynamic stall onset is directly related to the dynamic pressure lag, in agreement with previous Navier-Stokes simulations. Also, results are presented showing the effect of airfoil shape and Reynolds number on the onset of dynamic stall.

\section{Nomenclature}

$$
\begin{aligned}
& c=\text { chord length } \\
& C_{p}=\text { pressure coefficient, }\left(p-p_{\infty}\right) / q_{\infty} \\
& f=\text { frequency in } H z \\
& k=\text { for ramp motion: nondimensional pitch rate, } 2 \pi / \tau_{c} \\
& \quad \text { for oscillating motion: reduced frequency, } 2 \pi f c / V_{\infty} \\
& p=\text { pressure } \\
& p_{\infty}=\text { freestream pressure } \\
& q_{\infty}=\text { freestream dynamic pressure, } 1 / 2 \rho_{\infty} V_{\infty}^{2} \\
& R_{L}=\text { chord Reynolds number, } V_{\infty} c / \nu_{\infty} \\
& t=\text { time } \\
& U=\text { tangent velocity in the boundary layer } \\
& U_{e}=\text { boundary layer edge velocity } \\
& V_{\infty}=\text { freestream velocity } \\
& \alpha=\text { angle of attack, positive clockwise }
\end{aligned}
$$

This paper is declared a work of the U.S. Government and is not subject to copyright protection in the United States. 


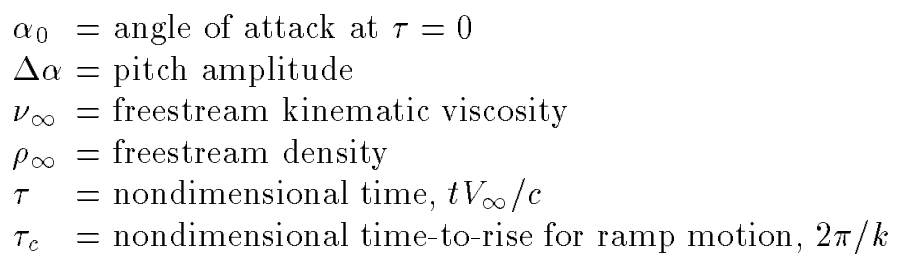

\section{Introduction}

Rapid incidence changes or unsteady airfoil motions are known to cause the phenomenon of dynamic stall. For flow moving from left to right this phenomenon involves the generation of a clockwise vortex that is produced by the large amount of coherent vorticity created near the airfoil's leading edge region. As the incidence angle is increased well past the static stall angle, the vortex begins to grow and convect over the suction surface. A survey of the available literature, for example the recent review paper of Carr and Chandrasekhara[1], reveals that the process of dynamic stall is strongly dependent on the airfoil geometry, especially on the leading-edge shape, Mach number, degree of flow unsteadiness or nondimensional pitch rate, state of the airfoil boundary layer, type of airfoil motion (pitch or plunge), location of pitch axis, etc. As a result of this dependence on many parameters, the computational prediction of dynamic stall using Navier-Stokes codes is a very time-consuming task and, as pointed out by Ekaterinaris and Platzer[2], is hampered by the need to include the proper modeling of the boundary-layer transition process in addition to adequate turbulence modeling. On the other hand, semi-empirical dynamic-stall models require little computational effort but they tend to obscure the major physical mechanisms which influence the dynamicstall process. Ericsson and Reding[3], in their review of the fluid dynamics of unsteady, separated flow, emphasized the need for the modeling of the proper physical phenomena, especially for the inclusion of time-lag effects due to circulation lag, boundary-layer convection lag, and lag due to moving flow separation points. In particular, they introduced the moving-wall or leading-edge jet effect by pointing out that an upward pitching airfoil creates fuller, even jet-like boundary layer profiles which therefore delay dynamic stall.

In this paper, we attempt to quantify the time-lag effects by using a combined panel and boundary-layer code for the analysis of unsteady, incompressible, attached airfoil flows with laminar, transitional or turbulent boundary layers. The use of such a code has the advantage of computational efficiency, but it limits the analysis to low-speed flows. Nevertheless, it enables the prediction of the onset of dynamic stall because, for many airfoils, the dynamic-stall process is initiated by the formation of a laminar separation bubble near the airfoil leading edge at a relatively low angle of attack. Hence predicting the onset of dynamic stall becomes a problem of predicting laminar flow separation.

In recent experiments, Chandrasekhara et al. [4] and Chandrasekhara and Carr [5] have provided conclusive evidence of the importance and magnitude of the time-lag effect caused by transiently or sinusoidally pitching airfoils. The effect 
of pitch rate or reduced frequency on the initiation of dynamic stall on a NACA 0012 airfoil in flows with freestream Mach numbers of 0.2 and 0.25 , as presented in Refs. 4 and 5, are shown in Figs. 1 and 2, respectively.

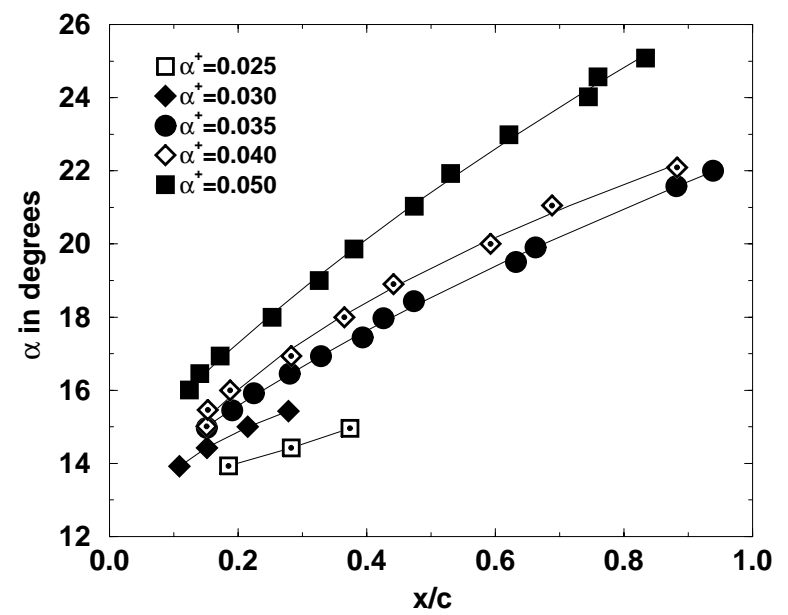

Figure 1. Dynamic-stall onset measurements at Mach 0.20 (Ref. 4.). The nondimensional pitch-rate is $\alpha^{+}=\dot{\alpha} c / V_{\infty}$ where $\dot{\alpha}$ is the pitch-rate in $\mathrm{deg} / \mathrm{s}$.

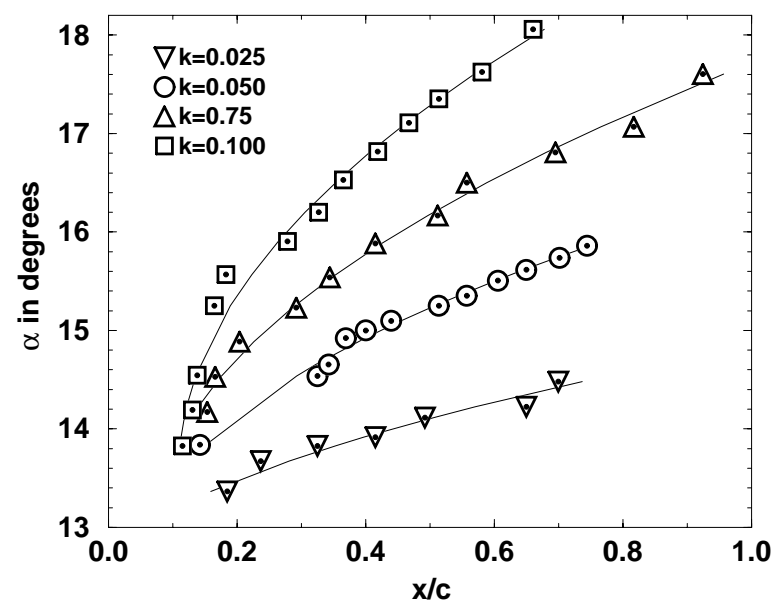

Figure 2. Dynamic-stall onset measurements at Mach 0.25 (Ref. 5).

Here the reduced frequency is given by $k=2 \pi c / V_{\infty}$.

The Reynolds number was 400,000 . It is seen that an increase in pitch rate or reduced frequency caused a delay in the dynamic stall vortex formation and propagation. It must be emphasized that these experiments were based on flowvisualization experiments using a stroboscopic Schlieren system. Therefore, the identification of the dynamic-stall vortex is subject to some error and, most im- 
portantly, the details of the dynamic-stall initiation process cannot be ascertained with such a method. In particular, the incidence angle at which a small separation bubble is formed cannot be determined, but it is obvious that it occurs at incidence angles which are much smaller than the angles plotted in Figs. 1 and 2.

The hybrid panel/boundary-layer code used in this paper allows the rapid and precise determination of the pressure distributions, pressure-lag effects and boundary-layer characteristics due to airfoil incidence changes, oscillation mode, frequency and amplitude, as well as the change of these characteristics with airfoil geometry. The major elements of this hybrid code are described in the next section, which is followed by a description of representative results.

\section{Approach}

The numerical methods utilized in the panel code and the boundary-layer code are briefly summarized in the following subsections, with details and validations of the methods available in the cited references.

\subsection{PANEL CODE}

Flow solutions are computed using an unsteady, potential-flow code originally developed by Teng [6]. The basic, steady panel code follows the approach of Hess and Smith [7], where the airfoil is approximated by a finite number of panels, each with a local, uniform, distributed source strength and all with a global, uniform, distributed vorticity strength. For $n$ panels there are $n$ unknown source strengths, $q_{j}$, and an unknown vorticity strength, $\gamma$. Boundary conditions include flow tangency at the midpoint of the $n$ panels and the Kutta condition which postulates that the pressure on the upper and lower surfaces of the airfoil at the trailing edge must be equal.

The unsteady panel code adopts the procedure of Basu and Hancock [8], where a wake panel is attached to the trailing edge through which vorticity is shed into the flow, as shown in Fig. 3.

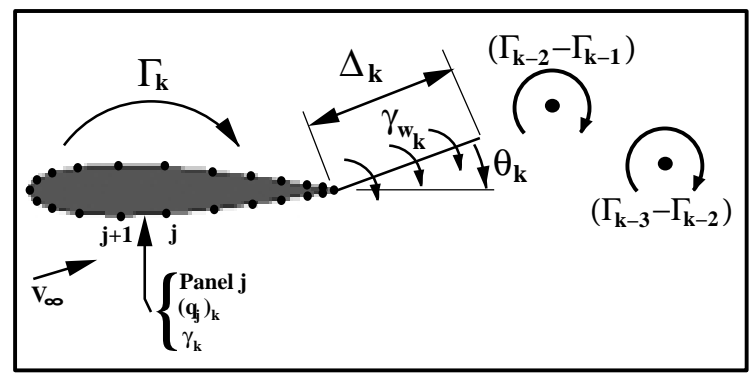

Figure 3. Schematic of the panel code wake model.

The Helmholtz theorem states that the bound vorticity in a flow remains constant, thus a change in circulation about the airfoil must result in the release 
of vorticity into the wake equal in magnitude and opposite in direction, given numerically by

$$
\Delta_{k}\left(\gamma_{W}\right)_{k}+\Gamma_{k}=\Gamma_{k-1}
$$

where $\Delta$ is the wake panel length, $\gamma_{W}$ is the distributed vorticity strength on the wake panel and $\Gamma$ is the circulation about the airfoil, and where the subscript $k$ indicates the current time step, and $k-1$ indicates the previous time step.

The wake panel introduces two additional unknowns; the wake panel length and its orientation, $\theta_{k}$. Thus, two additional conditions must be specified for closure;

1. The wake panel is oriented in the direction of the local resultant velocity at the panel midpoint.

2. The length of the wake panel is proportional to the magnitude of the local resultant velocity at the panel midpoint and the time-step size.

At the end of each time step the vorticity contained in the wake panel is concentrated into a point vortex which is shed into the wake and convected downstream with the flow, influencing and being influenced by the other shed vortices and the airfoil. Note, implementation of this approach requires an iterative scheme, since the velocity direction and magnitude used to define the wake panel are not initially known. Note also that this wake model is nonlinear. The unsteady panel code has been extensively documented in Refs. 6 and 9-14.

\subsection{BOUNDARY-LAYER CODE}

Flow properties in the boundary layer are computed using the Keller-Cebeci box method [15]. The code was generated and combined with a steady panel code by Nowak [16]. The general algorithm and several modifications used in the current implementation are discussed below.

The boundary layer code treats the airfoil surface as a flat plate with a variable pressure gradient, and steady conditions are assumed within the boundary layer. Laminar, transitional and turbulent regions are considered, and the turbulent region is computed using the Cebeci-Smith (CS) eddy-viscosity model. Like all eddy-viscosity methods, the CS model leaves the basic boundary layer equations unchanged but modifies the viscosity term by adding a local eddy viscosity, $\nu=$ $\nu_{\infty}+\epsilon_{m}$. The CS model divides the viscous region into an inner and an outer layer with the eddy viscosity in each region empirically formulated. The inner region is modeled by

$$
\left(\frac{\epsilon_{m}}{\nu}\right)_{i}=0.16 \sqrt{R e_{x}}[1-\exp (-y / A)]^{2} \eta^{2} \nu_{\infty} \gamma_{t r}
$$

and the outer region is modeled by

$$
\left(\frac{\epsilon_{m}}{\nu}\right)_{o}=0.0168 \sqrt{R e_{x}}\left[\eta_{e}-f_{e}\right] \gamma_{t r}
$$

where

$$
R e_{x}=\frac{U_{e}}{V_{\infty}} \xi R_{L}
$$




$$
\frac{y}{A}=\frac{\eta}{26} \sqrt[4]{R e_{x}} \sqrt{\nu_{w}}
$$

and where $\xi, \eta$ and $f$ are the Falkner-Skan variables. The term $\gamma_{t r}$ models the length of the transition or intermittency region, and its formulation is discussed below.

The point of transition onset is of critical importance for the prediction of dynamic stall. For the steady implementation of the code developed by Nowak the transition point is specified as input, presumably determined from experimental data. This is of little use in the present unsteady approach, as transition points would need to be specified for an infinite variety of conditions. Thus Michel's criterion is used to predict transition onset, where transition is initiated when the Reynolds number based on momentum thickness, $R_{\theta}$, and the Reynolds number based on $x, R e_{x}$, satisfy the equation

$$
R_{\theta_{t r}}=1.174\left(1+\frac{22,400}{R e_{x_{t r}}}\right) R e_{x_{t r}}^{0.46}
$$

where $R_{\theta}=U_{e} \theta / \nu_{\infty}$.

The Chen-Thyson intermittency model is used to predict the transition length where

$$
\gamma_{t r}=1-\exp \left[-G\left(x-x_{t r}\right) \int_{x_{t r}}^{x} \frac{1}{U_{e}} d x\right]
$$

and

$$
G=\frac{1}{G_{t r}}\left(\frac{U_{e}}{V_{\infty}}\right)^{3} R_{L}^{2} R_{x_{t r}}^{-1.34}
$$

In the original Chen-Thyson formulation $G_{t r}$ is set to a constant value of 1200 , but in the present implementation it is given by Cebeci [17] as a function of the transition Reynolds number

$$
G_{t r}=71\left[\ln \left(R e_{x_{t r}}\right)-4.732\right]
$$

The use of Michel's criterion for the prediction of transition onset and the use of the Chen-Thyson model for the transition region has been validated in Refs. 17 and 18 .

Note, that while the boundary-layer routine is steady, it has been shown that, for low reduced frequencies, changes in the boundary layer occur much more quickly than changes in the external flow, thus a steady boundary-layer analysis is sufficient [19].

The present combination of panel and boundary-layer codes is not an inviscid/viscous interaction approach (eg. Cebeci et al. [20]); that is, information is only passed from the panel code to the boundary-layer code; never the other way. Therefore, it is not possible to predict the viscous flow region beyond separation, 
as the flow separation would have a significant influence on the effective body shape seen by the panel code. However, the hybrid code can be used to predict the point in the cycle and on the airfoil surface where separation first occurs, and by looking in detail at the velocity profiles in the boundary layer, in particular in the region surrounding the suction peak, much insight may be obtained into the initial stages of dynamic stall. Note, the present code runs simulations in a matter of seconds on a workstation, and does not have convergence problems sometimes encountered with inviscid/viscous interaction methods.

\section{Results}

It is important to note that the only information the boundary layer algorithm is given is the surface point distribution and the external velocity distribution computed by the panel code at each time step. From the velocity distribution the local pressure and pressure gradient are computed, and it is really the pressure gradient that determines the nature of the boundary layer. Thus, it is essential to compute the surface pressure distribution accurately, and by looking at the pressure distributions, as well as the predicted position of the stagnation point, much can be determined about the flowfield without the aid of the boundary layer code.

As an additional consideration, note that in the unsteady panel code the pressure distribution is computed from the unsteady Bernoulli equation and depends on both the local velocity and the time-rate-of-change of the potential. In the boundary layer code this second term is not considered, but for the frequencies of interest in this study the term is negligible.

It is instructive to consider the pressure and boundary layer response of a NACA 0012 airfoil to a ramp change in angle of attack given by the function

$$
\alpha(\tau)=\alpha_{0}+\Delta \alpha\left[10\left(\frac{\tau}{\tau_{c}}\right)^{3}-15\left(\frac{\tau}{\tau_{c}}\right)^{4}+6\left(\frac{\tau}{\tau_{c}}\right)^{5}\right]
$$

where

$$
\tau_{c}=\frac{2 \pi}{k}
$$

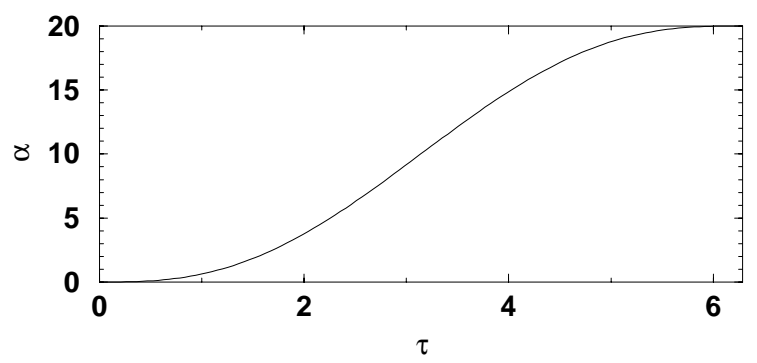

Figure 4. Modified-ramp motion; $0 \leq \alpha \leq 20$ degrees, $k=1$. 
This function is depicted in Fig. 4. The constant $k$ controls the pitch rate of the airfoil about the leading edge and hence the rate of incidence change. It is seen that the pressure build-up increasingly lags behind the steady-state pressure at a given angle of attack as the rate of incidence change, $k$, is increased. The pressure distributions in the vicinity of the leading edge are shown in Fig. 5 as a function of $k$ at an incidence of 4.53 degrees.

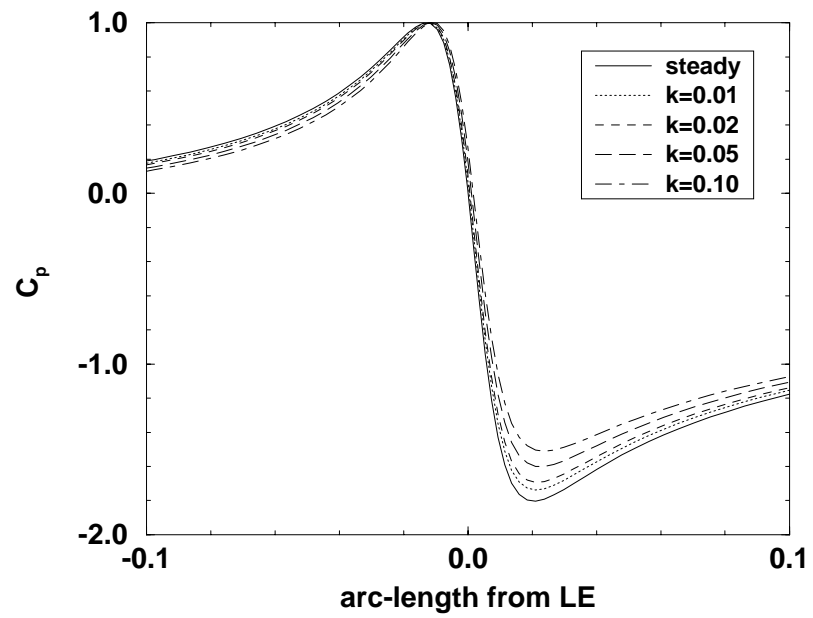

Figure 5. Pressure distribution near the LE.

The boundary-layer profiles at the same incidence angle of 4.53 degrees at a position 10 percent downstream from the leading edge on the suction surface are shown in Fig. 6. It is seen that the profiles are less developed as $k$ is increased.

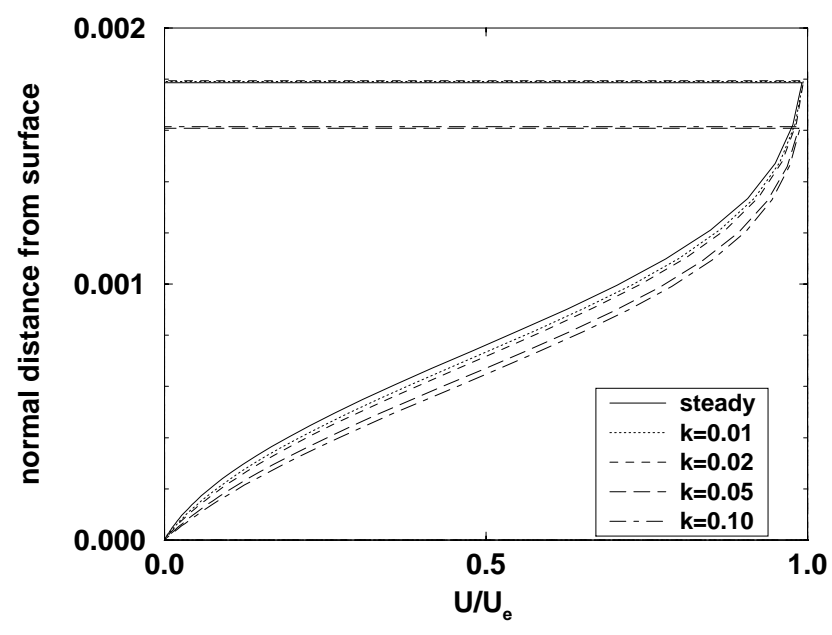

Figure 6. Boundary layer profiles. 
This is consistent with the lag in migration of the stagnation point, as shown in Fig. 7, as $k$ is increased because it produces a shorter running length for the boundary-layer build-up from the stagnation point to the 10 percent chord position.

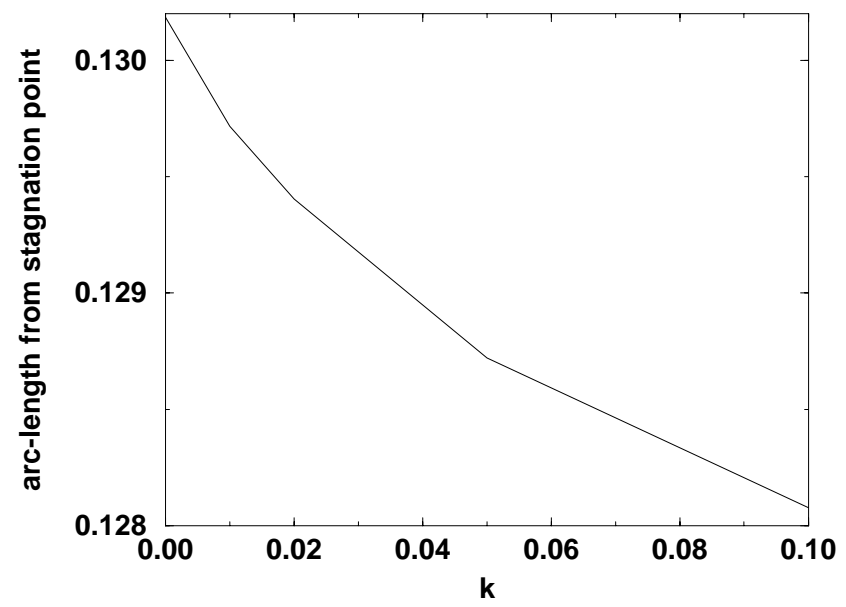

Figure 7. Distance from stagnation point to $0.1 c$.

Using the ramp change in angle of attack given by Eq. (10) between an initial value of 0 degree to a final value of 20 degrees it is possible to search for the incidence angle where laminar flow separation is first predicted. The dynamic stall onset angle for a NACA 0012 airfoil for a range of Reynolds numbers and frequencies is shown in Fig. 8.

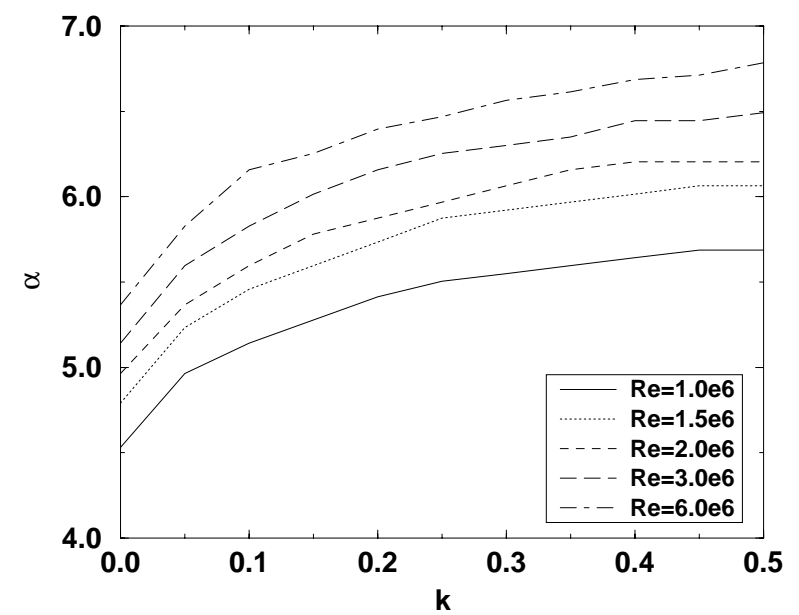

Figure 8. Stall onset AOA variation for a NACA 0012. 
As expected, laminar separation is delayed as the rate of incidence change is increased. The pressure distributions and the pressure gradient distributions, shown in Figs. 9 and 10 for a Reynolds number of 1 million, are virtually identical in each case. An increase in Reynolds number from 1 to 6 million delays the dynamic stall onset by approximately 1 degree.

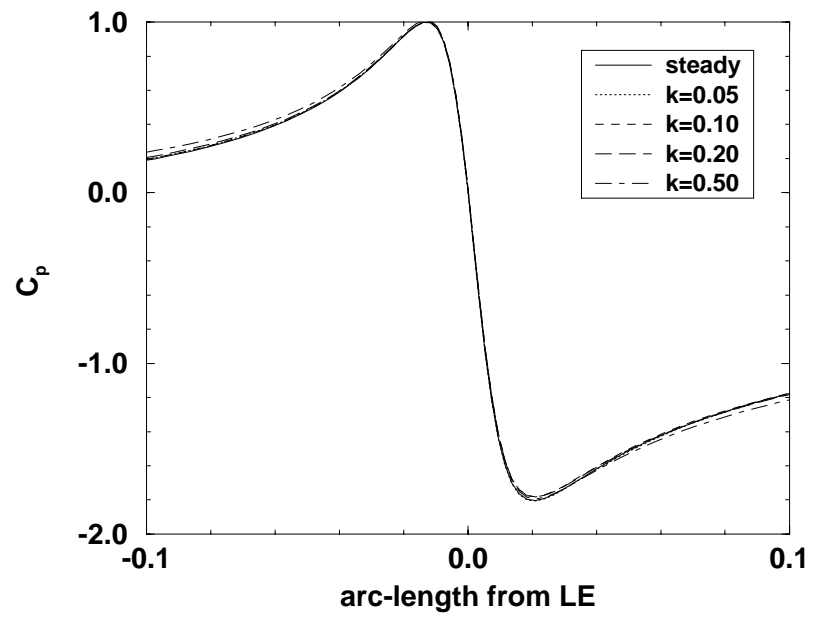

Figure 9. Pressure distribution near the LE at stall onset.

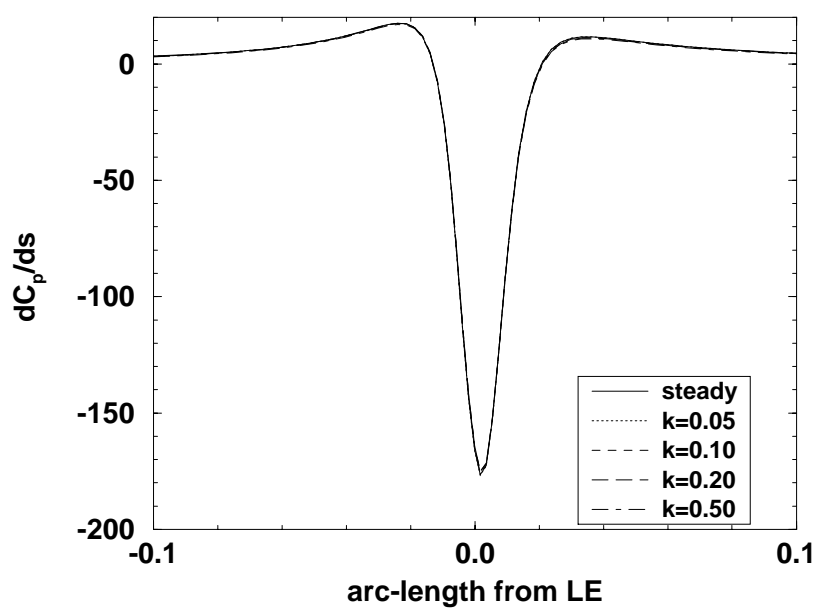

Figure 10. Pressure gradient distribution near the LE at stall onset.

A sharper leading edge reduces the dynamic stall onset angle, as shown in Fig. 11 for the NACA 0009 airfoil, whereas a rounder leading edge delays it, as depicted in Fig. 12 for the NACA 0015 airfoil. Additional results are shown in Figs. 13 and 14 for the NACA 1412 and 2412 airfoils. 


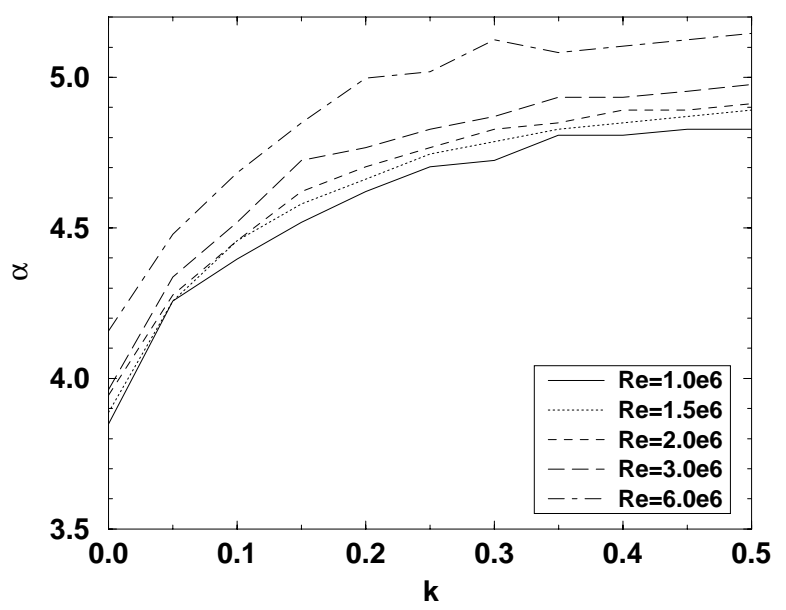

Figure 11. Stall onset AOA variation for a NACA 0009.

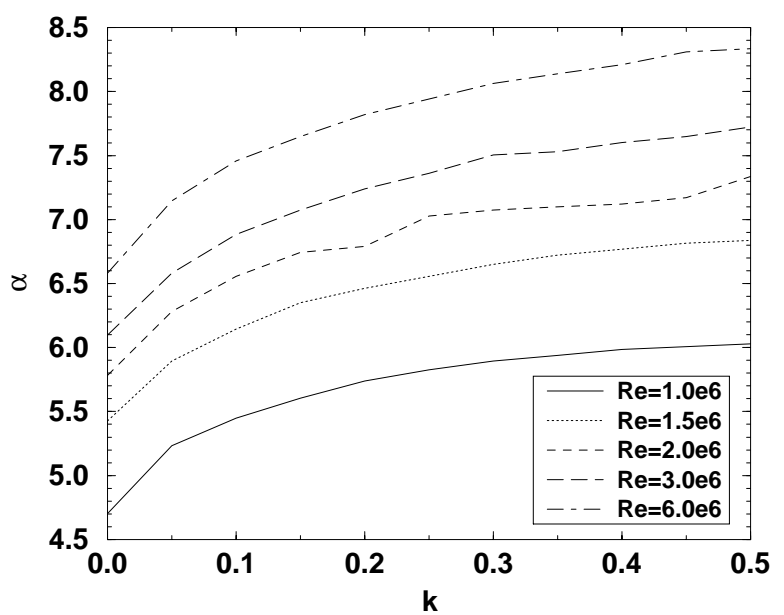

Figure 12. Stall onset AOA variation for a NACA 0015.

Ekaterinaris and Platzer [2] showed in their Navier-Stokes analysis of the dynamic stall behavior of a NACA 0012 airfoil which oscillates with an amplitude of 5 degrees about a mean angle of attack of 9 degrees in a low subsonic flow $(\mathrm{M}=0.3)$ of 4 million Reynolds number that it is essential to incorporate the boundary layer transition near the leading edge in order to obtain the experimentally measured lift, drag and moment hysteresis loops. It is instructive to study the boundarylayer development with the hybrid panel/boundary layer code and to compare with the Navier-Stokes calculations. This is shown in Fig. 15 where the onset of dynamic stall is again found to be delayed to a larger angle of attack in comparison to the steady-state stall onset if natural boundary-layer transition is assumed to occur. 


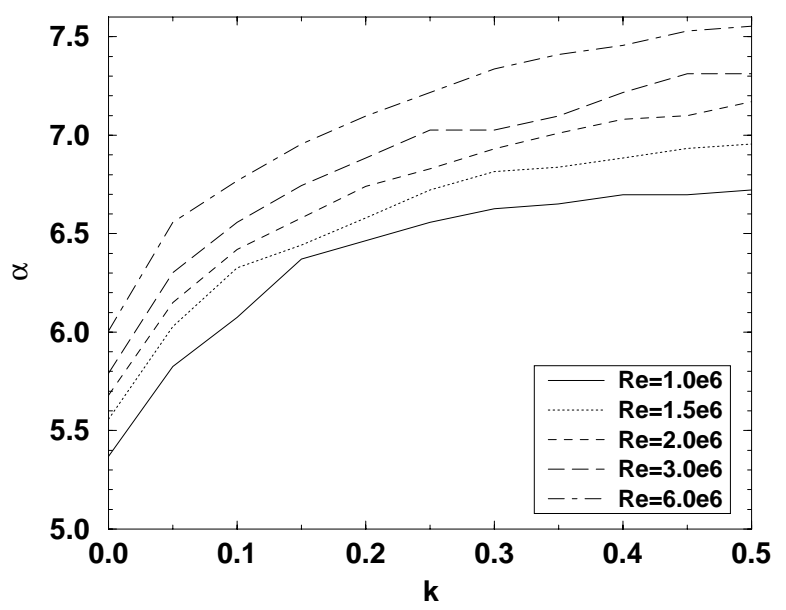

Figure 13. Stall onset AOA variation for a NACA 1412.

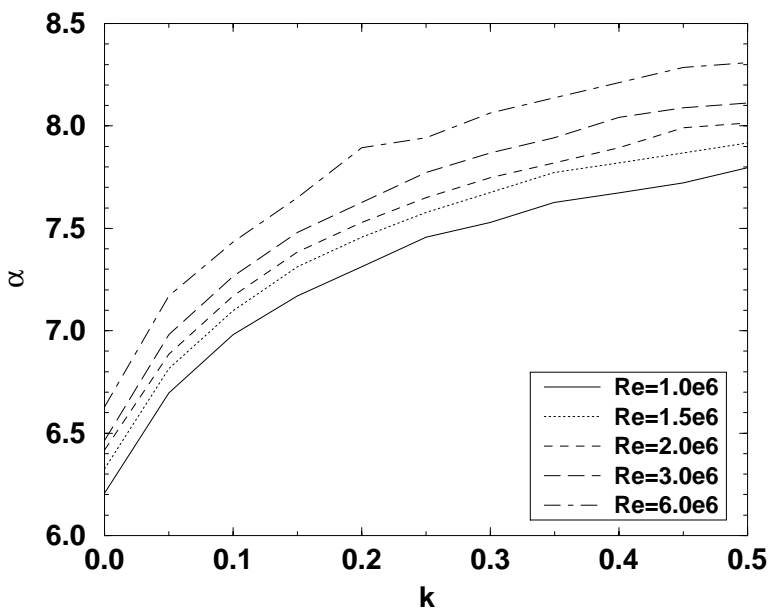

Figure 14. Stall onset AOA variation for a NACA 2412.

On the other hand, if the boundary layer is tripped into turbulent flow at the leading edge, the panel/boundary-layer code predicts turbulent separation from the trailing edge occurring it a much larger angle of attack. This is again in agreement with the fully turbulent Navier-Stokes calculations of Ref. 2.

A similar Navier-Stokes analysis has recently been carried out by Geissler et al. [21] for a NACA 0012 airfoil which oscillates in pitch with an amplitude of 10 degrees about a mean angle of attack of 10 degrees. For this case Chandrasekhara et al. [22] have provided experimental results. The Navier-Stokes computations again showed that it is crucial to account for the effect of boundary-layer transition. The fully turbulent Navier-Stokes computation predicts dynamic stall to occur at an incidence of about 17 degrees, whereas the transitional flow calculation puts it 
at about 14 degrees, in agreement with the measurement. The transitional hybrid code predicts dynamic-stall onset to occur at about 5 degrees, as shown in Fig. 16 , whereas a fully turbulent calculation delays it to about 18 degrees.

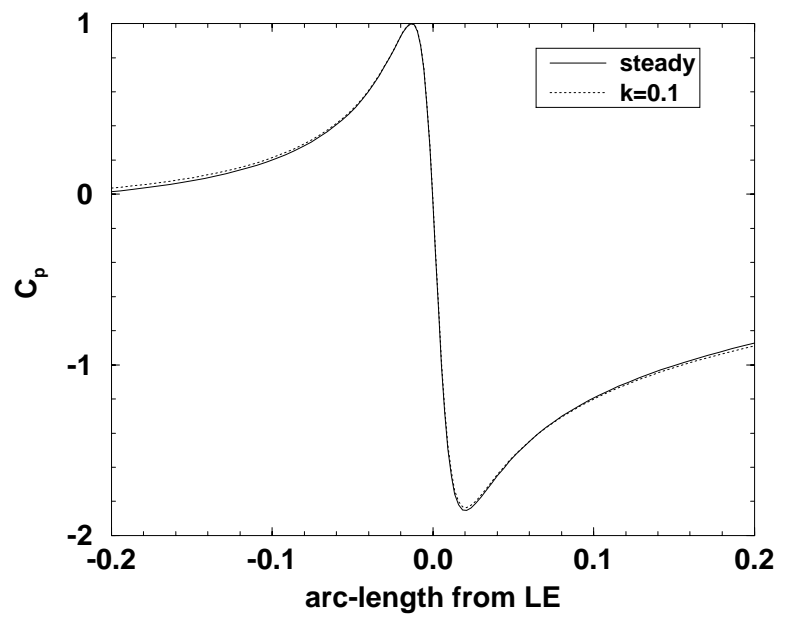

Figure 15. Pressure distribution at dynamic-stall onset for a sinusoidally pitching NACA 0012 airfoil; $\alpha=9 \pm 5$ degrees, $R e=4 \times 10^{6}$.

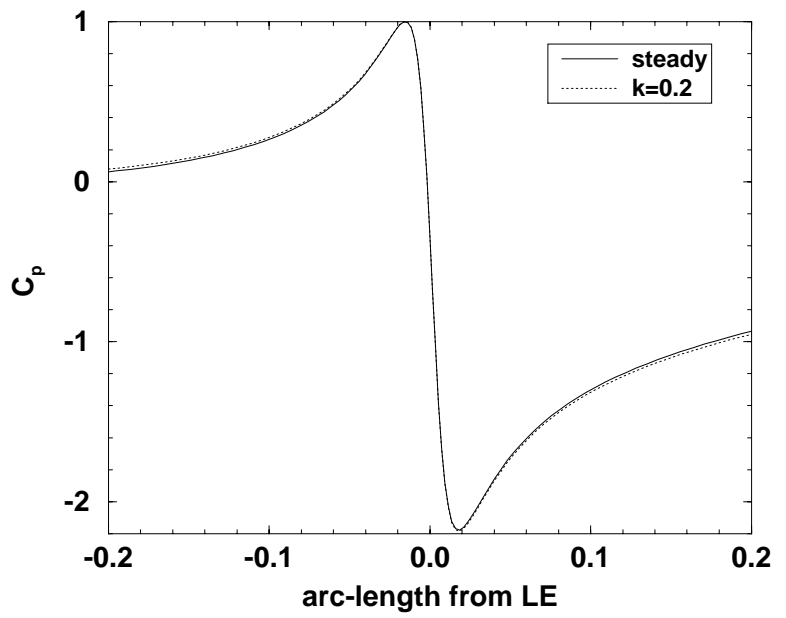

Figure 16. Pressure distribution at dynamic-stall onset for a sinusoidally pitching NACA 0012 airfoil; $\alpha=10 \pm 10$ degrees, Re $=1.1 \times 10^{6}$.

The incidence where stall onset is predicted by the hybrid code is plotted as a function of the reduced frequency in Figs. 17 and 18, corresponding to the configurations of Figs. 15 and 16, respectively. For these sinusoidally pitching calculations, typically several periods are required for the transients to fade and a periodic solution to form. The predicted stall angles are plotted for the first 
three cycles. The results for the first cycle are very much like the ramp motion, as expected, but the results during the later cycles are quite different and require further investigation.

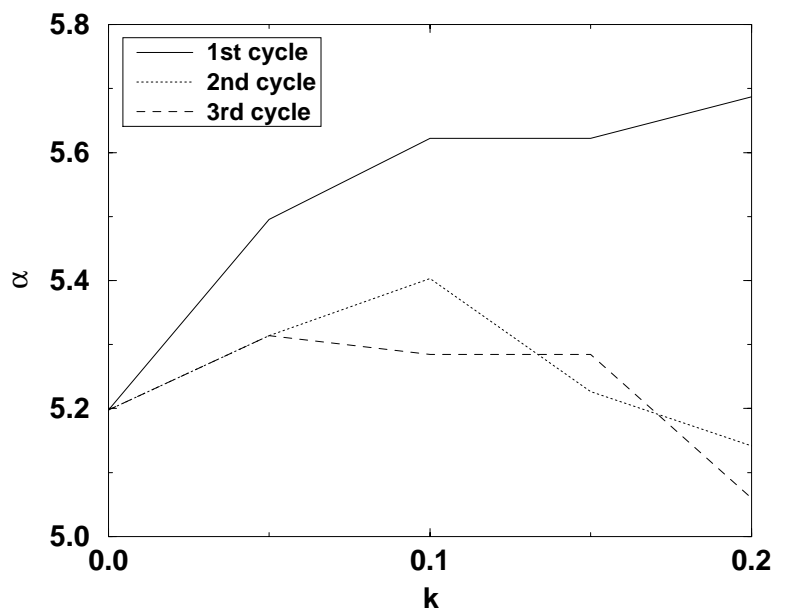

Figure 17. Incidence for dynamic-stall onset for a sinusoidally pitching NACA 0012 airfoil; $\alpha=9 \pm 5$ degrees, $R e=4 \times 10^{6}$.

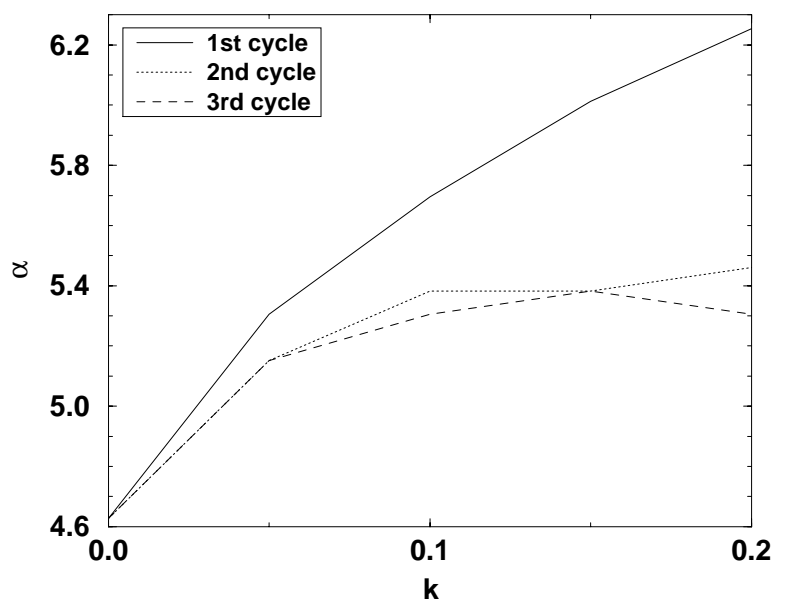

Figure 18. Incidence for dynamic-stall onset for a sinusoidally pitching NACA 0012 airfoil; $\alpha=10 \pm 10$ degrees, Re $=1.1 \times 10^{6}$.

Further results, showing the effect of oscillation mode (pitch versus plunge) on the pressure distribution and boundary-layer development, have recently been given by Jones and Platzer [23] using the hybrid panel/boundary-layer code. 


\section{Conclusions}

An efficient computational approach was presented for the fast prediction of the onset of dynamic stall due to rapid incidence changes or due to pitch / plunge motions of blades or airfoils. The method provides a means for the systematic evaluation of flow properties leading to dynamic stall over a broad parameter space.

Presented results suggest that the primary factor in dynamic stall onset is the phase lag in the surface pressure, data that may be obtained to the point of separation using a purely inviscid analysis. Viscous results obtained from the hybrid code illustrate that the pressure lag effect delays flow separation in dynamic cases even at very low frequencies, and that the onset of dynamic stall occurs when a critical pressure gradient distribution is obtained. Furthermore, in agreement with previous Navier-Stokes simulations [24], the critical pressure distribution leading to dynamic stall was found to be independent of mode and frequency.

\section{Acknowledgments}

This investigation was partially supported by the Naval Research Laboratory.

\section{References}

1. Carr, L.W. and Chandrasekhara, M.S., "Compressibility Effects on Dynamic Stall", Progress in Aerospace Sciences 32 (1996), 523-573.

2. Ekaterinaris, J.A. and Platzer, M.F., "Numerical Investigation of Stall Flutter", J. Turbomachinery 118-4 (1996), 197-203.

3. Ericsson, L.E. and Reding, J.P., "Fluid Dynamics of Unsteady Separated Flow, Part II, Lifting Surfaces", Progress in Aerospace Sciences 24 (1987), 249-356.

4. Chandrasekhara, M.S., Ahmed, S. and Carr, L.W., "Schlieren Studies of Compressibility Effects on Dynamic Stall of Transiently Pitching Airfoils", J. Aircraft 30-2 (1993), 213-220.

5. Chandrasekhara, M.S. and Carr, L.W., "Flow Visualization Studies of the Mach Number Effects on Dynamic Stall of an Oscillating Airfoil", J. Aircraft 27-6 (1990), 516-522.

6. Teng, N.H., "The Development of a Computer Code for the Numerical Solution of Unsteady, Inviscid and Incompressible Flow over an Airfoil," Master's Thesis, Naval Postgraduate School, Monterey, CA, June 1987.

7. Hess, J.L. and Smith, A.M.O., "Calculation of Potential Flow about Arbitrary Bodies," Progress in Aeronautical Sciences 8 (1966), 1-138.

8. Basu, B.C. and Hancock, G.J., "The Unsteady Motion of a Two-Dimensional Aerofoil in Incompressible Inviscid Flow," J. Fluid Mechanics 87 (1978), 159-168.

9. Platzer, M.F., Neace, K.S. and Pang, C.K., "Aerodynamic Analysis of Flapping Wing Propulsion," AIAA Paper No. 93-0484, Jan. 1993.

10. Riester, P.J., "A Computational and Experimental Investigation of Incompressible Oscillatory Airfoil Flow and Flutter Problems," Master's Thesis, Naval Postgraduate School, Monterey, CA, June 1993. 
11. Turner, M., "A Computational Investigation of Wake-Induced Airfoil Flutter in Incompressible Flow and Active Flutter Control," Master's Thesis, Naval Postgraduate School, Monterey, CA, March, 1994.

12. Tuncer, I.H., Platzer, M.F. and Ekaterinaris, J.A., "Computational Analysis of Flapping Airfoil Aerodynamics," ASME Fluids Engineering Division, Summer Meeting, June, 1994.

13. Jones, K.D. and Platzer, M.F., "Time-Domain Aeroelastic Analysis of a two Airfoil System with Application to Unsteady Rotary Wing Flowfields," AIAA Paper No. 95-0337, Jan., 1995.

14. Jones, K.D., Dohring, C.M. and Platzer, M.F., "Wake Structures Behind Plunging Airfoils: A Comparison of Numerical and Experimental Results," AIAA Paper No. 96-0078, Jan., 1996.

15. Keller, H.B. and Cebeci, T., "Accurate Numerical Methods for Boundary-Layer Flows, pt. 2, Two-Dimensional Turbulent Flows," AIA A Journal 10 (1972), 1193.

16. Nowak, L.M., "Computational Investigations of a NACA 0012 Airfoil in Low Reynolds Number Flows," Master's Thesis, Naval Postgraduate School, Monterey, CA, Sept., 1992.

17. Cebeci, T., "Essential Ingredients of a Method for Low Reynolds-Number Airfoils," AIA A Journal 27-12 (1989), 1680-1688.

18. VanDyken, R.D., Ekaterinaris, J.A., Chandrasekhara, M.S. and Platzer, M.F., "Analysis of Compressible Light Dynamic Stall Flow at Transitional Reynolds Numbers," A IA A Journal 34-7 (1996), 1420-1427.

19. Hancock, G.J. and Mabey, D.G., "Unsteady Aerodynamics of Controls," AGARD CP-465, April, 1990.

20. Cebeci, T., Platzer, M.F., Jang, H.M. and Chen, H.H., "An Inviscid-Viscous Interaction Approach to the Calculation of Dynamic Stall Initiation on Airfoils," J. Turbomachinery 115-4 (1993), 714-723.

21. Geissler, W., Chandrasekhara, M.S., Platzer, M.F. and Carr, L.W., "The Effect of Transition Modeling on the Prediction of Compressible Deep Dynamic Stall", Proc. 7th Asian Congress of Fluid Mechanics, Madras, India, Dec. 8-12, 1997.

22. Chandrasekhara, M.S., Wilder, M.C. and Carr, L.W., "Reynolds Number Influence on 2-D Compressible Dynamic Stall", AIAA Paper No. 96-0073, Jan. 1996.

23. Jones, K.D. and Platzer, M.F., "A Fast Method for the Prediction of Dynamic Stall Onset on Turbomachinery Blades," ASME Paper No. 97-GT-101, June 1997.

24. Grohsmeyer, S.P., Ekaterinaris, J.A. and Platzer, M.F., "Numerical Investigation of the Effect of Leading Edge Geometry on Dynamic Stall of Airfoils," AIAA Paper No. 91-1798, June, 1991. 